\title{
Bladder Cancer versus Hemorrhagic Cystitis: A Case of Mistaken Identity in a 34-Year-Old Male Undergoing Therapy for Granulomatosis with Polyangiitis
}

\author{
Jonathan Amatruda ${ }^{a} \quad K^{2}$ in Dieckhaus ${ }^{b}$ Poornima Hegde ${ }^{c}$ \\ John Taylor 3rd \\ ${ }^{a}$ University of Connecticut School of Medicine, and ${ }^{b}$ Division of Infectious Disease, \\ 'Department of Pathology, and d'Division of Urology, University of Connecticut Health \\ Center, Farmington, Conn., USA
}

\section{Key Words}

Hemorrhagic cystitis · BK virus · Polyomavirus · Immunosuppression · Granulomatosis with polyangiitis

\begin{abstract}
A 34-year-old male was referred for management of bladder cancer noted on workup for gross hematuria and new-onset irritative voiding symptoms. The patient's history was significant for recently diagnosed granulomatosis with polyangiitis for which he was undergoing treatment with oral cyclophosphamide and corticosteroids. Cystoscopy revealed lesions suspicious for malignancy, but the patient was diagnosed with hemorrhagic cystitis secondary to BK virus infection upon cytology review, and immunostaining confirmed a polyomavirus infection of the urothelium. The patient's symptoms resolved after a modification of his immunosuppressive regimen, and antiviral therapy was ultimately unnecessary. Though symptomatic BK virus infection of the genitourinary tract is common in immunosuppressed transplant patients, its occurrence in a patient undergoing immunomodulation for an autoimmune disease has not been reported yet. This case illustrates the potential for active BK virus infections in atypical patient populations and underscores the importance of rigorous hematuria workup, particularly in patients with multiple risk factors.
\end{abstract}




\section{Introduction}

Hemorrhagic cystitis (HC) is one of many causes of gross hematuria and may be incited by a variety of factors including chemotherapeutic agents (e.g. cyclophosphamide, ifosfamide), radiation therapy, or infection. BK virus-associated HC is a relatively rare diagnosis, typically reported in posttransplant patients whose immunosuppression permits viral reactivation and replicative infection [1]. We present a patient initially referred with the diagnosis of bladder cancer, who on further evaluation was reclassified as having HC secondary to BK polyomavirus infection. Our patient had been maintained on low-dose immunosuppression for granulomatosis with polyangiitis (previously known as Wegener's granulomatosis) with steroids and oral cyclophosphamide for $>1$ year. This case is a remarkable example of BK virus infection causing $\mathrm{HC}$ in a patient without the typical risk factors, and it underscores the value of a thorough medical history and workup of patients with hematuria.

\section{Case Report}

A 34-year-old man was referred for an evaluation of presumptive bladder cancer diagnosed on gross hematuria evaluation. The patient reported a sudden onset of lower urinary tract discomfort and burning associated with a mild end-stream discoloration of his urine. These symptoms progressed, and he was empirically treated for a bacterial urinary tract infection by his primary physician with two separate courses of antibiotics. His dysuria worsened and he noted the onset of severe urinary frequency associated with bloody urine and small clots. At this point, he was referred to a urologist who performed a standard hematuria workup including urinalysis, cystoscopy and CT of the abdomen and pelvis. Imaging was unremarkable for genitourinary pathology except for haziness of the perivesicular fat, which was suggestive of an inflammatory or infiltrative process. Urinalysis was positive for hemoglobin and a urine culture was negative. Cystoscopy revealed a mass-like effect on the anterior and posterior bladder walls with inflammation, erythematous lesions, bullous edema, and focal necrosis highly suspicious of bladder cancer. Urine cytology was notable for atypia. Based on these findings, the patient was referred to a tertiary center for management.

Further questioning revealed a medical history notable for never-smoking status and a diagnosis of granulomatosis with polyangiitis, which had been made 8 months prior. He had initially presented with skin lesions on the lower extremities and subsequent laboratory studies confirmed the diagnosis. Workup demonstrated an involvement of the lung, nasopharynx, eyes, and skin; however, the patient's kidney function was intact and preserved over the course of his illness and treatment. He underwent inpatient induction therapy with plasmapheresis and high-dose corticosteroids and was discharged on an immunosuppressive regimen of $15 \mathrm{mg}$ prednisone and $200 \mathrm{mg}$ cytoxan daily. Three months prior to presentation, his prednisone regimen was reduced to $12.5 \mathrm{mg}$ daily. Cystoscopy revealed findings similar to what has been previously noted (fig. 1). Cytology review revealed atypia consistent with polyomavirus infection. Biopsy was considered but deferred pending immunostain for polyomavirus (SV-40 antigen) on the previous urine cytology sample. SV-40 antigen immunostaining revealed a pattern of reactivity consistent with polyomavirus infection (fig. 2). With a preliminary diagnosis of HC secondary to BK polyomavirus, the patient was referred to an infectious disease specialist for management. Specific antiviral therapy was not initiated in favor of discontinuing cyclophosphamide and 
starting methotrexate for immunosuppression. Supportive measures were provided for symptoms which waned quickly and finally returned to baseline.

\section{Discussion}

Hematuria is associated with many medical diagnoses, some of which represent urologic diseases. While the most common presentation for bladder cancer is hematuria, the converse is not true. The assessment of patients with hematuria necessitates a thorough medical history and careful evaluation. The complexity of our case includes the diagnosis of granulomatosis with polyangiitis and ongoing therapy with cyclophosphamide and immunosuppression, all of which can lead to hematuria for different reasons.

Granulomatosis with polyangiitis is a form of vasculitis that affects small and mediumsized vessels of many organs. In $75 \%$ of cases, it involves the kidney and characteristically causes a rapidly progressive glomerulonephritis [2]. Gross hematuria is seldom seen in glomerulonephritis. Hematuria in this setting is microscopic resulting from intrarenal pathology secondary to inflammation and it is classically associated with red blood cell casts. Our patient's presentation is inconsistent with what would be expected from granulomatosis with polyangiitis-associated rapidly progressive glomerulonephritis. No red blood cell casts were observed on microscopic urinalysis nor was there any evidence of declining kidney function or proteinuria at any point in his treatment.

Initial therapy with 3-6 months of moderate-dose cyclophosphamide $(2 \mathrm{mg} / \mathrm{kg} / \mathrm{day})$ plus glucocorticoids is the standard of care for disease suppression and remission induction in granulomatosis with polyangiitis [3]. The use of cyclophosphamide is complicated by its toxicity, particularly in the genitourinary tract. Cyclophosphamide causes $\mathrm{HC}$ and exposure to cyclophosphamide significantly increases the risk for bladder cancer. Cyclophosphamideassociated $\mathrm{HC}$ is due to acrolein, a renally cleared metabolite that concentrates in the bladder, where it causes urothelial necrosis and inflammation [4, 5]. Cyclophosphamide toxicity is dose dependent and associated HC may present either acutely or chronically depending on the cumulative drug exposure over a given time period [6]. High-dose IV cyclophosphamide therapy is most likely to cause acute $\mathrm{HC}$, appearing within days of administration. Conversely, prolonged low-to-moderate doses of oral cyclophosphamide may lead to a chronic cystitis with symptoms appearing $\geq 1$ year after initiating therapy [6]. The risk of $\mathrm{HC}$ in patients undergoing cyclophosphamide therapy can be reduced by shortening the treatment course, fluid administration and coadministration of mesna (a chemical inactivator of acrolein). Dexamethasone may also be effective in reducing bladder inflammation due to cyclophosphamide. However, dexamethasone was not found to be effective on its own and initial administration of mesna is required to protect against the development of HC [4]. At the time of symptom onset, our patient's cumulative cyclophosphamide intake was 30-40 g over $<7$ months - a level of exposure which has not been implicated in either acute or chronic forms of cyclophosphamide-associated HC.

While urologists are keenly sensitive to the relationship between blood in the urine and bladder cancer, this is an unusual diagnosis in a 34-year-old with no smoking history, in spite of his cyclophosphamide exposure. Only $0.4-1.5 \%$ of all bladder tumors present in this age group with a $0.02 \%$ probability of representing invasive disease $(1$ in 4,942$)$ [7]. Cohort studies of patients treated with cyclophosphamide have consistently shown a dosedependent increased risk for bladder cancer, and there is at least some evidence of an increased baseline risk for granulomatosis with polyangiitis patients even without cyclophosphamide exposure [8-10]. However, the duration of low-dose cyclophosphamide 
treatment in our patient was relatively short and not considered predisposing for the development of bladder cancer. A study of cyclophosphamide-associated bladder cancers reported that cancer diagnoses followed the initiation of therapy by at least a few years [10]. Our patient's cyclophosphamide dose represents a risk factor for bladder cancer later in his life and warrants a low threshold for workup. However, the short interval between the initiation of therapy and the onset of the patient's symptoms are uncharacteristic of cyclophosphamide-associated bladder cancer.

Given the patient's age, his relatively modest and brief exposure to cyclophosphamide, and the cytolopathologic findings, the proximate cause of $\mathrm{HC}$ was suggested to be a BK virus reactivation due to immunosuppressive therapy for granulomatosis with polyangiitis. BK virus is a human polyomavirus known to cause significant renal and urinary tract disease in immunosuppressed patients. Though HC secondary to BK virus infection is well-described in transplant patients undergoing intense immunosuppression, it is an uncommon problem outside of this setting [11]. Furthermore, BK virus reactivation in a patient receiving immunomodulation to treat autoimmune disease has not been previously described.

BK virus is a member of the papovavirus family, which also includes JC virus and SV-40. BK virus is found in virtually all populations but does not cause significant disease in the immunocompetent host. A recent report shows that up to $86.3 \%$ of hospitalized patients have plyomavirus viruria, with a higher prevalence in those without versus those with asymptomatic hematuria [12]. When host defenses are significantly suppressed, latent viruses may reactivate and initiate a lytic life cycle which manifests clinically as disease of the tissues in which the virus has reactivated [11]. BK virus has a tropism for the urinary tract, and common complications of reactivation in immunocompromised hosts include HC, nephritis, and nephropathy. BK virus is ubiquitous in humans, with documented seroprevalence rates of up to $90 \%$ by late childhood [13]. A clinically significant infection is most frequently seen in patients receiving renal and hematopoietic stem cell transplants, in which case the profound immunosuppression required for successful engraftment sets the stage for virus reactivation. BK virus infections are relatively infrequent among patients undergoing solid organ transplantation except in the case of renal grafts, which carry a 1-10\% risk of infection in the posttransplant period [1,14]. Currently, there is no widely accepted explanation for the increased risk of BK virus in renal transplant patients compared to recipients of other solid organs. Bennett et al. [11] hypothesized that renal transplant represents a compound risk of viral reactivation due to immunosuppression and local tissue injury [15]. Symptomatic BK virus infection has been reported in patients with AIDS and other states of immunocompromise, but these cases are exceptional. BK virus reactivation due to immunosuppressive therapy used to manage autoimmune disease is unprecedented in the literature but offers insight into the management of these patients.

Methods for diagnosing BK virus infection demonstrate the presence of the virus either directly by PCR and immunohistochemistry, or indirectly by cytologic examination for characteristic viral cytopathic changes (e.g. decoy cells) [14]. Decoy cells are about twice the size of deep transitional cells and have scanty cytoplasm, sometimes with short cytoplasmic tails (comet cells). Their enlarged nuclei show characteristic large round, opaque, smudgy inclusions, which often artifactually leach out (fig. 2). Serology is not useful due to the high rate of exposure and ubiquity of latent infection. PCR for viral DNA in urine or plasma is relatively quick and noninvasive but lacks sensitivity; an observation of decoy cells on urine cytology is more sensitive but less specific [14]. The gold standard diagnostic procedure is biopsy with histological analysis for cytopathic effects in addition to a positive polyomavirus/SV-40 immunostain. In order to maximize accuracy and minimize invasiveness, we 


\begin{tabular}{l|l}
\hline DOI: $10.1159 / 000363692$ & $\begin{array}{l}\text { C 2014 S. Karger AG, Basel } \\
\text { www.karger.com/cru }\end{array}$ \\
\hline
\end{tabular}

Amatruda et al.: Bladder Cancer versus Hemorrhagic Cystitis: A Case of Mistaken Identity in a 34-Year-Old Male Undergoing Therapy for Granulomatosis with Polyangiitis

elected to forgo biopsy but increased the specificity by performing urine cytology for decoy cells as well as immunohistochemistry using polyomavirus/SV-40 (polyclonal) antibody.

Studies of BK virus infection in renal transplant patients indicate that the level of immunosuppression predicts the risk of BK-induced $\mathrm{HC}$ more than the type of immunosuppressive agent $[11,16]$. Our patient had been on dual immunomodulator therapy with cyclophosphamide and prednisone, and his risk of BK-associated HC may have been increased by subclinical urothelial injury from concurrent cyclophosphamide therapy. Some investigators have reported circumstantial evidence that cyclophosphamide therapy before hematopoietic stem cell transplant is associated with the development of BK virus $\mathrm{HC}$ after transplantation [15]. This process in the bladder may be analogous to that occurring in the renal grafts.

In conclusion, this appears to be the first description of symptomatic BK virus infection in a patient undergoing immunosuppressive treatment for granulomatosis with polyangiitis. This case is instructive for the clinical assessment of patients presenting with hematuria. The diagnosis of BK virus-induced HC could be made only by the careful evaluation of the patient's history, symptoms, laboratory data, imaging, and pathological findings. The identification of BK virus-associated $\mathrm{HC}$ in a patient who presented with none of the classic epidemiological risk factors demonstrates that this condition may appear in an outpatient urological setting and should not be ruled out prematurely.

\section{References}

1 Garces JC: BK virus-associated nephropathy in kidney transplant recipients. Ochsner J 2010;10:245-249.

2 Seo P, Stone JH: The antineutrophil cytoplasmic antibody-associated vasculitides. Am J Med 2004;117:3950.

-3 Langford CA: Update on the treatment of granulomatosis with polyangiitis (Wegener's). Curr Treat Options Cardiovasc Med 2012;14:164-176.

4 Morais MM, Belarmino-Filho JN, Brito GAC, Ribeiro RA: Pharmacological and histopathological study of cyclophosphamide-induced hemorrhagic cystitis - comparison of the effects of dexamethasone and Mesna. Braz J Med Biol Res 1999;32:1211-1215.

5 Cox PJ: Cyclophosphamide cystitis - identification of acrolein as the causative agent. Biochem Pharmacol 1979;28:2045-2049.

-6 Hu RQ, Mehter H, Nadasdy T, Satoskar A, Spetie DN, Rovin BH, Herbert L: Severe hemorrhagic cystitis associated with prolonged oral cyclophosphamide therapy: case report and literature review. Rhe umatol Int 2008;28:1161-1164.

7 Howlader N, Noone AM, Krapcho M, Garshell J, Neyman N, Altekruse SF, Kosary CL, Yu M, Ruhl J, Tatalovich Z, Cho H, Mariotto A, Lewis DR, Chen HS, Feuer EJ, Cronin KA (eds): SEER Cancer Statistics Review, 19752010, National Cancer Institute. Bethesda. http://seer.cancer.gov/csr/1975_2010/, based on November 2012 SEER data submission, posted to the SEER web site, April 2013.

-8 Travis LB, Curtis RE, Glimelius B, Holowaty EJ, van Leeuwen FE, Lynch CF, Hagenbeek A, Stovall M, Banks PM, Adami J, Gospodarowicz MK, Wacholder S, Inskip PD, Tucker MA, Boice JD Jr: Bladder and kidney cancer following cyclophosphamide therapy for non-Hodgkin's lymphoma. J Natl Cancer Inst 1995;87:524-530.

$\$ 9$ McCarroll N, Keshava N, Cimino M, Chu M, Dearfield K, Keshava C, Kligerman A, Owen R, Protzel A, Putzrath R, Schoeny R: An Evaluation of the mode of action framework for mutagenic carcinogens case study: cyclophosphamide. Environ Mol Mutagen 2008;49:117-131.

$>10$ Knight A, Aslding J, Granath F, Sparen P, Ekbom A: Urinary bladder cancer in Wegener's granulomatosis: risks and relation to cyclophosphamide. Ann Rheum Dis 2004;63:1307-1311.

11 Bennett SM, Broekma NM, Imperiale MJ: BK polyomavirus: emerging pathogen. Microbes Infect 2012;14:672-683.

$\checkmark 12$ Lee SH, Hong SH, Lee JY, Hwang TK, Kim KS, Lee H, Choi YJ: Asymptomatic hematuria associated with urinary polyomavirus infection in immunocompetent patients. J Med Virol 2014;86:347-353.

$\$ 13$ Knowles WA: Discovery and epidemiology of the human polyomaviruses BK virus (BKV) and JC virus (JCV). Adv Exp Med Biol 2006;577:19-45.

14 Hariharan S: BK virus nephritis after renal transplantation. Kidney Int 2006;69:665-662.

15 Harkensee C, Vasdev N, Gennery AR, Willetts IE, Taylor C: Prevention and management of BK-virus associated haemorrhagic cystitis in children following haematopoietic stem cell transplantation - a systematic review and evidence-based guidance for clinical management. Br J Haematol 2008;142:717-731. 


\begin{tabular}{l|l}
\hline Case Rep Nephrol Urol 2014;4:120-125 \\
\hline DOI: 10.1159/000363692 & $\begin{array}{l}\text { @ 2014 S. Karger AG, Basel } \\
\text { www.karger.com/cru }\end{array}$ \\
\hline
\end{tabular}

Amatruda et al:: Bladder Cancer versus Hemorrhagic Cystitis: A Case of Mistaken Identity in a 34-Year-Old Male Undergoing Therapy for Granulomatosis with Polyangiitis

16 Ramos E, Drachenberg CB, Wali R, Hirsch HH: The decade of polyomavirus BK-associated nephropathy: state of affairs. Transplantation 2009;87:621-630.

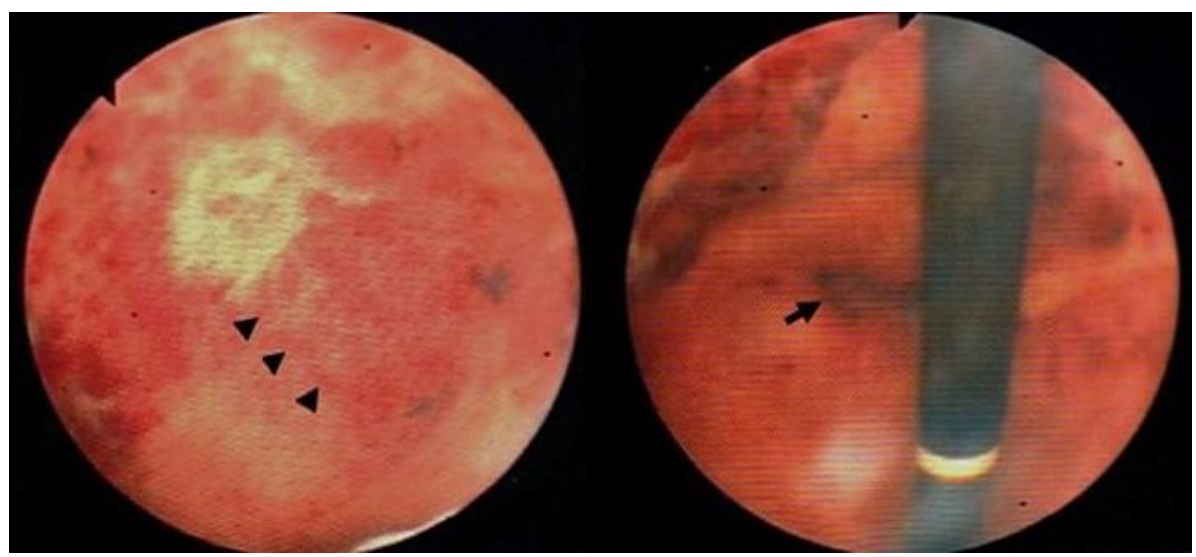

Fig. 1. Cystoscopy images showing bladder wall inflammation with bullous edema (arrowheads) and areas of necrosis (arrow) secondary to active BK virus infection.

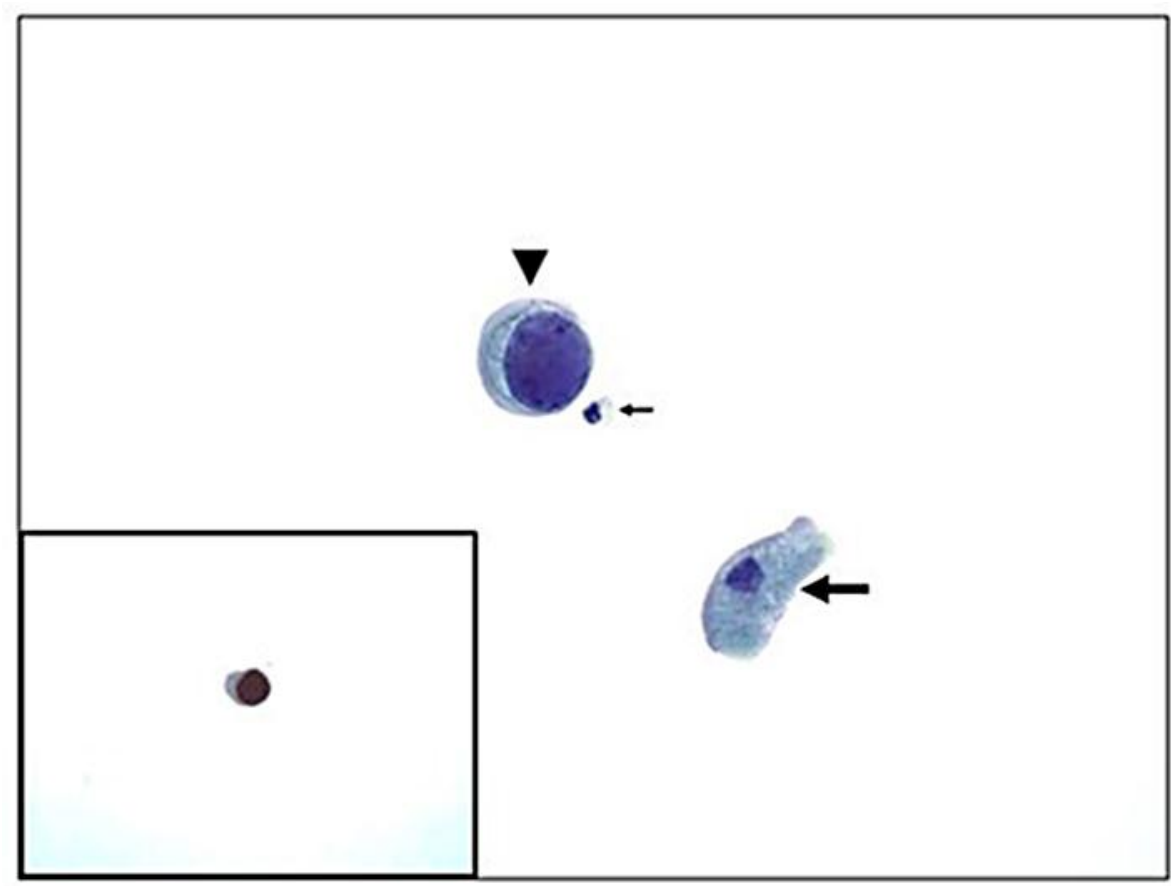

Fig. 2. Papanicolaou stain of a decoy cell (arrowhead) with a homogeneous smudgy nuclear inclusion and scanty cytoplasm. Size comparison with a normal urothelial cell (large arrow) and a neutrophil (small arrow) in the background. $\times 1,000$. Inset: Polyomavirus/SV-40 (polyclonal) antibody shows positive nuclear staining of viral inclusion. $\times 600$. 